\title{
Luminol Encapsulated Liposome as a Signal Generator for the Detection of Specific Antigen-antibody Reactions and Nucleotide Hybridization
}

\author{
Pakavadee Rakthong,* Akarin Intaramat,** and Kavi RatanabanangKoon****† \\ *Department of Microbiology, Faculty of Science, Mahidol University, Bangkok 10400, Thailand \\ **Chulabhorn Research Institute and Chulabhorn Graduate Institute, Bangkok 10210, Thailand
}

\begin{abstract}
Liposomes prepared with biotinylated phospholipids and luminol entrapped were shown to be of $187 \mathrm{~nm}$ in size, $59 \%$ of which were unilamellar and with $43 \%$ luminol trapping efficiency. Liposome prepared from biotinylated phospholipids with a longer hydrophilic PEG2000 spacer, but not with the shorter hydrophobic caproyl one, bound efficiently and specifically with immobilized streptavidin in a microplate assay. The interactions of dinitrophenol and tobramycin with their respective antibodies, and the hybridization of 20-mers oligonucleotides were studied using the liposome as a signal generator. These reactions were shown to be specific with limits of detection of $0.58 \mu \mathrm{M}, 0.96 \mu \mathrm{M}$ and $18 \mathrm{nM}$, respectively.
\end{abstract}

(Received March 3, 2010; Accepted May 10, 2010; Published July 10, 2010)

\section{Introduction}

Liposomes are vesicles in which an aqueous volume is enclosed by lipid bilayer. The use of liposomes as analytical and bio analytical reagents in both homogeneous and heterogeneous assays has been shown to be successful in a variety of applications because of their high surface area, large internal volume, and ability to conjugate bilayer lipids with many kinds of bio recognition molecules, for example, antibodies, nucleic acid, receptor and small molecules. ${ }^{1-4}$

Fluorescent markers are most commonly used for the detection of biomolecules because they can be measured easily and very sensitively. ${ }^{3,5}$ However, depending on the fluorescent molecules used the background can be high, thus in some cases chemiluminescence can become a marker of choice to achieve higher sensitivity of detection. Chemiluminescence is advantageous over fluorescence for its higher sensitivity, wider dynamic range; also, there is no need for an excitation lamp or expensive instrumentation. Although the combined use of liposomes with chemiluminescence has been reported, ${ }^{6,7}$ there have been only a few reports on the encapsulation of chemiluminescent molecules in liposomes. ${ }^{8,9}$ One of the most commonly used chemiluminescent molecules is luminol, which has been applied to a very broad field including immunoassay, ${ }^{10,11}$ non-immunoassay diagnostic ${ }^{12,13}$ and monitoring techniques as well as biosensors. ${ }^{14,15}$ The supposed emitter of luminol is the excited 3-aminophthalate anion with maximum emission at $425 \mathrm{~nm}^{16}$

There are two methods for coupling biomolecules to a liposome surface, which include covalent ${ }^{17,18}$ and noncovalent reactions..$^{19,20}$ The noncovalent methods, which may be based

$\dagger$ To whom correspondence should be addressed.

E-mail: sckrt@mahidol.ac.th; kavi@cri.or.th on the reaction of Protein A (for binding to antibodies) ${ }^{20}$ or of streptavidin (for binding to biotinylated molecules), ${ }^{19,21}$ provide a milder reaction condition. Streptavidin is a tetrameric protein and binds very tightly to biotin. The biotin-streptavidin dissociation constant of about $10^{-15} \mathrm{M}$ is among the highest known for biochemicals. One streptavidin molecule contains 4 biotin binding sites, two of which are on one side, while the other two are on the opposite side. Thus, streptavidin can serve as a linker to couple biotinylated biomolecules e.g., oligonucleotides, ${ }^{22}$ or antibodies ${ }^{19,21}$ to biotinylated liposomes.

The aim of the present study was to investigate the feasibility of using biotinylated luminol encapsulated liposome as a signal generator of specific antigen-antibody reactions and oligonuclotide hybridization. This study was performed based on a microtiter plate format.

\section{Experimental}

Reagents and chemicals

1,2-Dipalmitoyl-sn-glycero-3-phosphocholine (DPPC); 1,2-dipalmitoyl-sn-glycero-3-[phospho-rac-(1-glycerol)] (sodium salt) (DPPG); 1,2-dipalmitoyl-sn-glycero-3phosphoethanolamine (DPPE); 1,2-dipalmitoyl-sn-glycero-3phosphoethanolamine- $N$-(cap biotinyl) (sodium salt) (biotinylcap-PE); and 1,2-distearoyl-sn-glycero-3-phosphoethanolamine$N$-[biotinyl(polyethylene glycol) 2000] (ammonium salt)

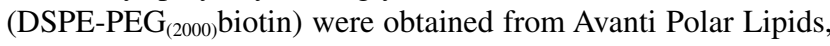
Inc. (Alabaster, AL). Cholesterol, luminol (5-amino-2,3dihydrophthalazine-1,4-dione), sodium deoxycholate (DOC), and para-aminobenzoic acid (PABA) were obtained from SigmaAldrich Co. (St. Louis, MO). Isopore ${ }^{\mathrm{TM}}$ membrane filters of $0.4-$ and $0.2-\mu \mathrm{m}$ pore sizes were purchased from Millipore (Ireland). Streptavidin was obtained from Nacalai Tesque, Inc. (Kyoto, Japan). Anti-dinitrophenyl monoclonal antibody 
(Anti-DNP $\mathrm{Ab}$ ) and biotin-X 2,4-dinitrophenyl-X-L-lysine succinimidyl ester (DNP-X biocytin-X,SE) were obtained from Invitrogen Corporation (Carlsbad, California). DNP-glycine was obtained from Fisher Scientific Company (NJ). Oligonucleotides were obtained from Genscript Corporation (Piscataway, NJ). Tobramycin and biotinamidocaproate Tobramycin amide (biotinylated-Tobramycin) were obtained from Toronto Research Chemicals (Canada), anti-Tobramycin antibody (Anti-Tobramycin $\mathrm{Ab}$ ) was obtained from Mybiosource (CA). Rabbit anti-Ecstasy antibody (Anti-Ecstasy Ab) was prepared in our laboratory.

\section{Preparation of biotinylated, luminol encapsulated liposomes}

Liposomes were prepared by a modification of the dehydrationrehydration method described by Martorell et al. ${ }^{23}$ in the following manner. Lipids that included DPPC, cholesterol and DPPG at $1.26,1.23$, and $0.13 \mu \mathrm{mol}$, respectively, were placed in a 50-ml Erlenmeyer flask. DPPE and biotinyl-Cap-DPPE, weighing 0.56 and $0.18 \mathrm{nmol}$, respectively, were also placed in the same flask. One milliliter of chloroform was added into the flask and shaken to make a homogeneous mixture of lipids. When the mixture was completely solubilized, chloroform was removed by evaporation at $45^{\circ} \mathrm{C}$ under a flow of nitrogen gas to give a lipid film. The lipid film was thoroughly dried under vacuum overnight to remove residual chloroform, and was then dispersed in $1 \mathrm{ml}$ of $0.1 \mathrm{M}$ Tris- $\mathrm{HCl}, \mathrm{pH} 8.6$ containing $100 \mathrm{mM}$ luminol. The mixture was vortexed at $45^{\circ} \mathrm{C}$ until all lipid film peeled off the side of glass surface to form multilamellar vesicles (MLVs). The MLVs were extruded 10 times through $0.4-\mu \mathrm{m}$, then 10 times through $0.2-\mu \mathrm{m}$ polycarbonate filters (Isopore $^{\mathrm{TM}}$, Membrane Filters), respectively, using a Miniextruder (Avanti Polar Lipids, Inc., Alabaster, USA) to produce particle of homogeneous size. The temperature of the extrusion device was maintained at $45^{\circ} \mathrm{C}$ throughout the procedure. Liposomes were dialyzed at $4^{\circ} \mathrm{C}$ against 4 changes of 100 vols. of $0.1 \mathrm{M}$ Tris- $\mathrm{HCl} \mathrm{pH} 8.6$ containing $0.15 \mathrm{M} \mathrm{NaCl}$ to remove unencapsulated luminol.

\section{Characterization of liposomes}

Size distribution. The particle size distribution and polydispersity of the suspended vesicles was determined by dynamic light scattering (Zetasizer nano ZS90, Malvern Instruments Ltd., Worcestershire, UK) at the National Nanotechnology Center (National Science and Technology Development Agency, Thailand).

Lamellarity. The lamellarity of the liposomes was studied under a transmission electron microscope (TEM) at the Center of Nanoimaging (Faculty of Science, Mahidol University) using a phosphotungstic acid negative staining method.

Chemiluminescent signal measurement. Under the optimal conditions at room temperature, the concentration of luminol was determined from a standard curve of chemiluminescent (CL) activity vs. luminol in the presence of 1.7 fold diluted empty liposome, $0.015 \% \mathrm{H}_{2} \mathrm{O}_{2}$ and $5 \%$ DOC, in $0.1 \mathrm{M}$ sodium carbonate buffer $\mathrm{pH} 10.0$. The CL signal, which remained stable over a period of at least $15 \mathrm{~min}$, was measured at $1 \mathrm{~min}$ $(t=1)$. The standard curve was linear in the range of $0.6-4.8 \mu \mathrm{mol} / \mathrm{l}$ with a correlation coefficient $\left(r^{2}\right)$ of 0.991 . The relative standard deviation (RSD) of the sample was $6.9 \%$ $(n=3)$. The unit of the measurement was in counts per minute (cpm).

Encapsulation efficiency. Thirty microliters of $0.1 \mathrm{M}$ Tris- $\mathrm{HCl}$, $\mathrm{pH} 8.6$ containing $0.15 \mathrm{M} \mathrm{NaCl}$ with or without DOC were added into a 96-well microplate. It was then followed by adding $50 \mu \mathrm{l}$ of a 1:1000 diluted undialyzed liposome suspension.
Fifty microliters of $\mathrm{H}_{2} \mathrm{O}_{2}$ at a concentration of $0.04 \%$ (v/v) were added to initiate the reaction. The encapsulation efficiency was calculated using the following formula: ${ }^{24}$

$$
\% \text { Chemiluminescent release }=\left[\left(C_{\mathrm{t}}-C_{0}\right) / C_{\mathrm{t}}\right] \times 100 \text {. }
$$

Here, $C_{\mathrm{t}}$ is the chemiluminescent signal from the wells with DOC and $C_{0}$ is the chemiluminescent signal from the wells without the detergent. The concentration of luminol was read from a standard curve. Wells containing mixtures of liposomes and DOC, liposomes and $\mathrm{H}_{2} \mathrm{O}_{2}$, and DOC and $\mathrm{H}_{2} \mathrm{O}_{2}$ were set as controls. Moreover, wells with either buffer, liposomes, DOC or $\mathrm{H}_{2} \mathrm{O}_{2}$ were also set as controls. The chemiluminescent signal from the well containing the mixture of luminol and $\mathrm{H}_{2} \mathrm{O}_{2}$ was set as a positive control. The chemiluminescence signal was measured as described above.

Studies on the reactivity of biotin on the surface of biotinylated liposomes

Determination of the biotin-binding activity of streptavidin in solution. The biotin binding activity of streptavidin was studied using a method modified from that of Der-Balian et al. ${ }^{25}$ A $20 \mu \mathrm{g} / \mathrm{ml}$ streptavidin solution was titrated with biotin at concentrations of $0,0.01,0.05,0.1,0.3,0.5$, and $0.75 \mu \mathrm{g} / \mathrm{ml}$ in a total volume of $300 \mu \mathrm{l}$ of $0.2 \mathrm{M}$ ammonium carbonate buffer $\mathrm{pH}$ 8.9. The absorbance change at $233 \mathrm{~nm}$ was measured on a Shimadzu Model UV-1700 double-beam recording spectrophotometer. The sample cuvette was zeroed at $233 \mathrm{~nm}$ against the buffer in the reference cuvette. The break point (point of change in slope) was estimated visually from the graph. Separate lines were drawn through the data points to the left and right of the break point. The intersection on these two lines was used to calculate the moles of biotin required to reach the break point.

Studies on the effect of spacers between biotin and phospholipid on the binding of biotinylated liposomes to immobilized streptavidin. A microtiter plate was coated with streptavidin at $10 \mu \mathrm{g} /$ well in a coating buffer $(50 \mu \mathrm{l}$ of sodium carbonate buffer $\mathrm{pH}$ 9.6) and incubated in a moist chamber at $4{ }^{\circ} \mathrm{C}$ overnight. The plate was then washed with a Tween washing solution (TWS, $0.05 \%$ Tween 20 in $0.15 \mathrm{M} \mathrm{NaCl}$ ) four times, and then blocked with $1 \%$ BSA in phosphate buffered saline (PBS) at room temperature for $1 \mathrm{~h}$. After four washes of the plate, biotinylated liposomes prepared using $0.5 \mathrm{~mol} \%$ of either DSPE-PEG $_{(2000)}$ biotin or biotinyl-Cap-DPPE in $0.1 \mathrm{M}$ Tris- $\mathrm{HCl}$ $\mathrm{pH} 8.6$ containing $0.15 \mathrm{M} \mathrm{NaCl}$, were added and the plate was incubated for $4 \mathrm{~h}$ at $4^{\circ} \mathrm{C}$. The plate was washed four times with $0.15 \mathrm{M} \mathrm{NaCl}, 0.1 \mathrm{M}$ Tris-HCl, pH 8.6. The levels of plate-associated liposomes were determined after adding DOC and $\mathrm{H}_{2} \mathrm{O}_{2}$ at final concentrations of 5 and $0.015 \%$, respectively, in $0.1 \mathrm{M}$ sodium carbonate buffer, $\mathrm{pH} 10.0$. The chemiluminescent signal of luminol was determined using an automated microplate reader (Wallac Victor ${ }^{2}$ 1420, Perkins Elmer, Finland). Each microtiter plate experiment was performed in triplicate.

Determination of the binding capacity of DSPE-PEG $G_{(2000)}$ biotinliposomes to immobilized streptavidin

A microtiter plate was coated with streptavidin at a concentration of $10 \mu \mathrm{g} /$ well in the coating buffer and incubated at $4{ }^{\circ} \mathrm{C}$ overnight in a moist chamber. The plate was then washed with TWS four times, and then blocked with $1 \%$ BSA in PBS at room temperature for $1 \mathrm{~h}$. After four washes, $50 \mu \mathrm{l}$ of biotinylated liposomes, prepared with various mol\% $(0,0.1,0.5$ and 1) of DSPE-PEG $(2000)$ biotin, and encapsulating $100 \mathrm{mM}$ of 
luminol were added, and the plate was then incubated for $4 \mathrm{~h}$ at $4^{\circ} \mathrm{C}$. The plate was washed with $0.15 \mathrm{M} \mathrm{NaCl}, 0.1 \mathrm{M}$ Tris- $\mathrm{HCl}$, $\mathrm{pH} 8.6$ four times. The levels of plate-associated liposomes were determined as described above. The non-specific binding of liposomes to the plate was determined by estimating the levels of biotinylated liposomes bound when incubated in the presence of excess free biotin (200 molar excess to the biotinylated lipids derivatives on liposome). The plate was washed by $0.15 \mathrm{M} \mathrm{NaCl}, 0.1 \mathrm{M}$ Tris- $\mathrm{HCl}, \mathrm{pH} 8.6$ four times. The levels of plate-associated liposomes were determined as described above.

\section{Determination of the specific binding of biotinylated liposomes} to streptavidin coated on microtiter plate

A microtiter plate was coated with streptavidin at $10 \mu \mathrm{g} / \mathrm{well}$, washed and blocked with BSA as described above. Free biotin solutions at concentrations of $0,1.67 \times 10^{-4}, 1.67 \times 10^{-3}$, $1.67 \times 10^{-2}$, or $1.67 \times 10^{-1} \mu \mathrm{g} / \mu \mathrm{l}$ were added to streptavidincoated wells for $5 \mathrm{~min}$, followed by adding biotinylated liposomes, and were then incubated for $4 \mathrm{~h}$ at $4^{\circ} \mathrm{C}$. Each plate was washed with $0.15 \mathrm{M} \mathrm{NaCl}, 0.1 \mathrm{M}$ Tris- $\mathrm{HCl}, \mathrm{pH} 8.6$ four times. The levels of plate-associated liposomes were determined as described above.

Studies on the interaction of antibody and antigen based on luminol encapsulated liposomes

The interactions of DNP and Tobramycin with their respective antibodies were used as models in the present study. A schematic diagram of the experiments is shown in Fig. S1-A (Supporting Information). Biotinylated DNP was prepared as follows. DNP-X biocytin-X,SE was hydrolyzed with $0.1 \mathrm{M}$ $\mathrm{NaOH}$ overnight to remove the succinimidyl ester, and then neutralized with $0.1 \mathrm{M} \mathrm{HCl}$. The biotinylated DNP was kept at $-20^{\circ} \mathrm{C}$ until used.

The anti-DNP antibody at a concentration of $1.88 \mu \mathrm{g} / \mathrm{well}$ in a coating buffer $(50 \mathrm{mM}$ sodium carbonate buffer $\mathrm{pH} 9.6)$ was passively immobilized on a microtiter plate and incubated overnight in a moist chamber at $4{ }^{\circ} \mathrm{C}$. The plate was washed with TWS four times, and then blocked with $1 \%$ BSA in PBS at room temperature for $1 \mathrm{~h}$. Biotinylated DNP in PBS containing $0.1 \%$ BSA (diluent) at a concentration of $0.31 \mu \mathrm{g} /$ well was added in the presence of either PABA or DNP-glycine, and incubated for $1 \mathrm{~h}$. The plate was washed 4 times with TWS. A streptavidin solution at concentration of $10 \mu \mathrm{g} / \mathrm{well}$ in diluent was added to the wells, and was incubated for $1 \mathrm{~h}$ at room temperature. The plate was washed with TWS, then biotinylated liposomes in $0.15 \mathrm{M} \mathrm{NaCl}, 0.1 \mathrm{M}$ Tris- $\mathrm{HCl} \mathrm{pH} 8.6$ were added to the wells, and the plate was incubated for $4 \mathrm{~h}$ at $4{ }^{\circ} \mathrm{C}$. The plate was washed 4 times with the $0.15 \mathrm{M} \mathrm{NaCl}, 0.1 \mathrm{M}$ Tris-HCl. The levels of plate-associated liposomes were determined as described above. Moreover, the specificity of the interaction was also studied by the binding of biotinylated DNP to other immobilized antibodies i.e., anti-Ecstasy and antiTobramycin antibodies under the same condition.

Similar to the experiment described above, anti-Tobramycin antibody at a concentration of $10 \mu \mathrm{g} /$ well was used instead of anti-DNP antibody for testing the interaction between Tobramycin and its antibody. In order to demonstrate the specific binding of this system, biotinylated Tobramycin at concentration of $5 \mu \mathrm{g} /$ well was used instead of biotinylated DNP, and was added in the presence of Tobramycin or other drugs (spectinomycin, kanamycin or amplicilin). The remaining procedure was the same as those of the DNP system described above.
Table 1 Oligonucleotide sequences

\begin{tabular}{lcc}
\hline \multicolumn{1}{c}{ Sample } & Modification & Sequence \\
\hline (+) ONT probe & 5'biotin $^{\prime}$ & 5'-GCACCAGCAGAATAACTGAG-3' \\
(-) ONT probe & $5^{\prime}$ 'biotin & 5'-CTCAGTTATTCTGCTGGTGC-3' \\
(+) ONT sample & - & 5'-GCACCAGCAGAATAACTGAG-3' \\
Mismatched & - & 5'-ACCTGTAACCTAGATGGCTT-3' \\
ONT & & \\
\hline
\end{tabular}

Studies on the hybridization of oligonucleotides based on the luminol encapsulated liposomes

The sequences of oligonucleotides are shown in Table 1. A schematic representation of the methods studied is shown in Fig. S1-B (Supporting Information). A microtiter plate was coated with streptavidin at a concentration of $10 \mu \mathrm{g} / \mathrm{well}$ in the $50 \mathrm{mM}$ PBS and incubated at $4{ }^{\circ} \mathrm{C}$ overnight. The plate was washed with $50 \mathrm{mM}$ PBS, and then blocked with $2 \%$ BSA in $50 \mathrm{mM}$ PBS at room temperature for $1 \mathrm{~h}$. After four washes of the plate with $50 \mathrm{mM}$ PBS, a $50-\mu \mathrm{l}$ volume of prewarmed $\left(45^{\circ} \mathrm{C}\right)$ biotinylated oligonucleotide $((+)$ ONT probe $\left.)\right)$ in $0.1 \mathrm{M}$ Tris, $1 \mathrm{mM}$ EDTA buffer, pH 8.0 (TE buffer) was added and incubated for $1 \mathrm{~h}$ at $37^{\circ} \mathrm{C}$. An unbound (+) ONT probe was removed by 4-time washings with TE buffer. Fifty microliters of a prewarmed $\left(55^{\circ} \mathrm{C}\right)$ biotinylated (-) ONT probe, either in the absence or presence of mismatched ONT or synthetic (+) ONT (Table 1) in TE buffer were added and incubated for $1 \mathrm{~h}$ at $52^{\circ} \mathrm{C}$. The unbound oligonucleotide was removed by washing 4 times with TE buffer. Streptavidin at a concentration of $10 \mu \mathrm{g} / \mathrm{well}$ was added and incubated for $1 \mathrm{~h}$ at room temperature. Then, biotinylated liposomes in $0.15 \mathrm{M} \mathrm{NaCl}, 0.1 \mathrm{M}$ Tris- $\mathrm{HCl}$ $\mathrm{pH} 8.6$ containing EDTA were added to the wells, and the plate was incubated for $4 \mathrm{~h}$ at $4^{\circ} \mathrm{C}$. The plate was washed 4 times with the TE buffer. The levels of plate-associated liposome were determined as described above.

\section{Results and Discussion}

Characterization of luminol encapsulated liposomes using dynamic light scattering

The liposome prepared as described showed an average size of $187 \mathrm{~nm}$ with a polydispersity index of 0.13 (data not shown) which was considered to be a homogeneous liposome suspension. Under TEM, 59\% of the 120-prepared liposomes were single unilamellar vesicles, while the remaining were of varying lamellarities. The preparation of luminol encapsulated liposomes resulted in $43 \%$ of luminol being entrapped.

Studies on the reactivity of biotinyl groups on the surface of biotinylated liposomes

Determination of the biotin-binding activity to streptavidin in solution. The binding of free biotin to free streptavidin causes a shift and an increase in the absorption spectrum of tryptophan at $233 \mathrm{~nm}$; when all of the binding sites were saturated with biotin, no further increase at $233 \mathrm{~nm}$ was detected. The titration curve (data not shown) gave a breaking point at a biotin concentration of $0.3 \mu \mathrm{g} / \mathrm{ml}$. The molar ratio of biotin to streptavidin at this point was 3.26:1, i.e., each mole of streptavidin bound 3.26 moles of biotin under this condition.

The effect of spacers between biotin and phospholipid on the binding of biotinylated liposomes to immobilized streptavidin. Two different biotinylated phospholipids were used to prepare biotinylated luminol entrapped liposomes. These were 


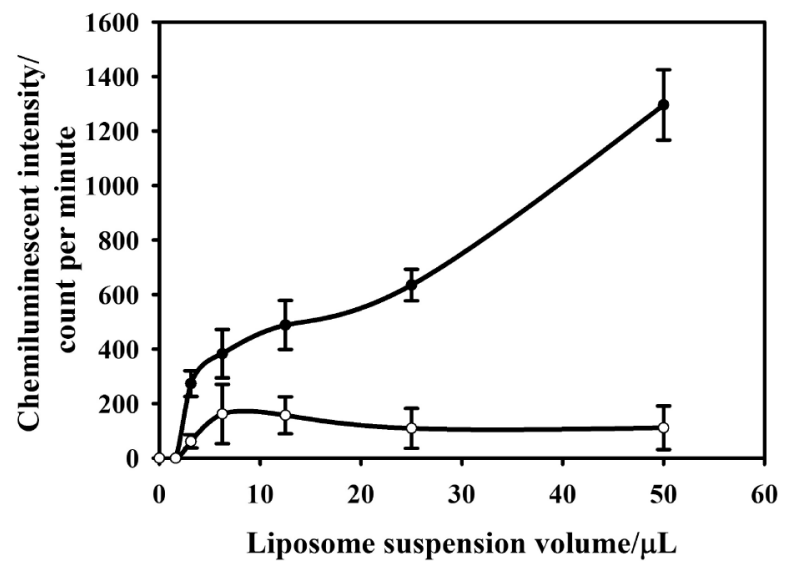

Fig. 1 Binding of luminol entrapped liposomes prepared with different biotinylated phospholipids to immobilized streptavidin. $\bullet$, DSPE-PEG ${ }_{(2000)}$ biotin liposomes; $\odot$, biotinyl-Cap-DPPE liposomes.

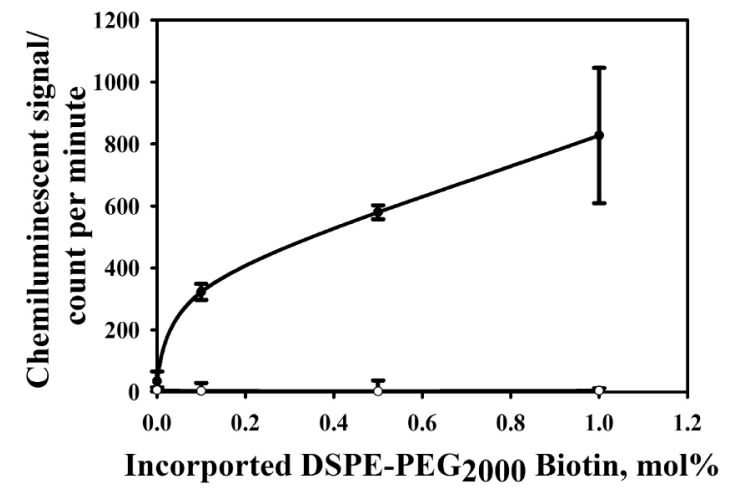

Fig. 2 Effect of the ligand density of DSPE-PEG (2000) $_{\text {biotin }}$ incorporated in liposomes on the binding of the liposomes to streptavidin immobilized on a microtiter plate and inhibition of the binding by free biotin. •, Absence of free biotin; $\odot$, presence of free biotin.
DSPE-PEG $_{(2000)}$ biotin or DPPE-Cap biotin, which were different in the size and chemical nature of the spacers used. The binding of liposomes containing either of these lipids to coated streptavidin was determined (Fig. 1). DSPE-PEG $(2000)$ biotin liposome gave a significant signal, while there was a very low chemiluminescent signal when liposomes prepared with biotinyl-Cap-DPPE were tested. The binding of biotinylated liposomes to immobilized streptavidin depends on the lipid compositions, ${ }^{26-28}$ chemical nature ${ }^{29}$ and length of spacers. ${ }^{26-28,30}$

A model described for the effect of a spacer on the binding of the biotinylated liposome to streptavidin has been purposed. ${ }^{30-32}$ According to this model, equilibrium exists between two states of hapten residues on the surface of liposomes, $\mathrm{H}_{\mathrm{m}}$ and $\mathrm{H}_{\mathrm{s}}$. $\mathrm{H}_{\mathrm{m}}$ represents a physical state of the hapten group that interacts with lipid membrane, and is not available for binding. $\mathrm{H}_{\mathrm{s}}$ represents that the group extends from the membrane, and can bind with other molecules. In the $\mathrm{H}_{\mathrm{m}}$ state, the hydrophobic interaction between a longer spacer and the lipid bilayer may be stronger than that of a shorter or intermediate spacer. That is, a hapten group with longer spacers becomes unavailable for binding, while haptens with very short spacers are less effective for binding due to steric constrains. ${ }^{30}$ It was therefore suggested that the intermediate chain length and hydrophilic spacers are more effective; thus a caproyl $\left(\mathrm{C}_{6}\right)$ spacer has been used with some degree of success. ${ }^{23,26,28}$

Based on the $\mathrm{C}-\mathrm{C}$ bond length in ethane of $0.15 \mathrm{~nm}$, the length of the caproyl spacer in its extended configuration is about $0.15 \times 5=0.75 \mathrm{~nm}$. With the $1 \mathrm{~nm}$-depth of biotin binding site on streptavidin, ${ }^{33}$ biotin attached to the caproyl spacer should not bind effectively to immobilized streptavidin. The hydrophilic and globular $\mathrm{PEG}_{2000}$ spacer $(6.5 \mathrm{~nm})^{34}$ should render biotin more exposed, and this should result in more effective conjugation. This was shown to be the case in the present study; liposome prepared using biotinyl-Cap-DPPE bound very poorly to streptavidin, but liposome containing DSPE-PEG ${ }^{2000}$ biotin bound effectively to streptavidin.

Effect of the ligand density and the specificity of binding of DSPE-PEG $G_{(2000)}$ biotin-liposomes to immobilized streptavidin

The effect of the ligand density of biotin on the liposome surface on the binding to immobilized streptavidin was studied. The liposome binding was found to be dependent on the amount

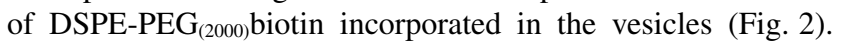

Liposomes prepared without biotinylated lipids ( $0 \mathrm{~mol} \%$ DSPE-PEG $_{(2000)}$ biotin) did not bind to immobilized streptavidin. The rate of increase of the signal-to-noise ratio declined at a very high ligand density. This indicates that a further increase in the ligand coverage to increase the reactivity of liposome is unlikely.

The binding of liposomes prepared with DSPE-PEG (2000) $_{\text {biotin }}$ to immobilized streptavidin was shown to be specific when free biotin was added to the reaction mixture. It was found that the binding level was decreased with increasing amount of free biotin, and reached the base-line level at $1.67 \mathrm{ng} / \mu \mathrm{l}$ of free biotin, which was equivalent to a $2 \times$ molar excess of biotin on the liposome surface (data not shown). No binding of biotinylated liposomes was observed in the presence of a 200-fold molar excess of free biotin (Fig. 2).

Luminol encapsulated liposomes as a signal generator in the study of antigen-antibody interaction and oligonucleotide hybridization

The interaction of biotinylated DNP with immobilized antiDNP antibody could be detected by a chemiluminescent reaction of biotinylated, luminol encapsulated liposomes (Fig. 3). The specific interaction between anti-DNP antibody and biotinylated DNP was determined by hapten inhibition by DNP-glycine. PABA had no effect on the binding. Moreover, the specific interaction was also demonstrated by the failure of biotinylated DNP to bind to other immobilized antibodies such as anti-Ecstasy and anti-Tobramycin antibodies (Fig. 3). The limit of detection (LOD), defined as the concentration (of competitor molecule) that inhibited $10 \%$ of the specific reaction, ${ }^{35}$ of DNP-glycine was $0.58 \mu \mathrm{M}$.

In a similar experiment, biotinylated luminol encapsulated liposomes were studied using anti-Tobramycin antibody and biotinylated Tobramycin (Fig. 4). The specific interaction was demonstrated by the competition of other aminoglycosides (kanamycin and spectinomycin) and a $\beta$-lactam (amplicilin) antibiotics (Fig. 4). Because of a structural similarity, other aminoglycosides can interfere with the specific interaction of the anti-Tobramycin antibody with biotinylated Tobramycin at different degrees. $\mathrm{IC}_{50}$ of Tobramycin was $3 \mu \mathrm{M}$, which is 100- and 1333-times lower than those of spectinomycin and kanamycin, respectively. No cross reactivity was observed with amplicilin. The LOD of Tobramycin was $0.96 \mu \mathrm{M}$. 


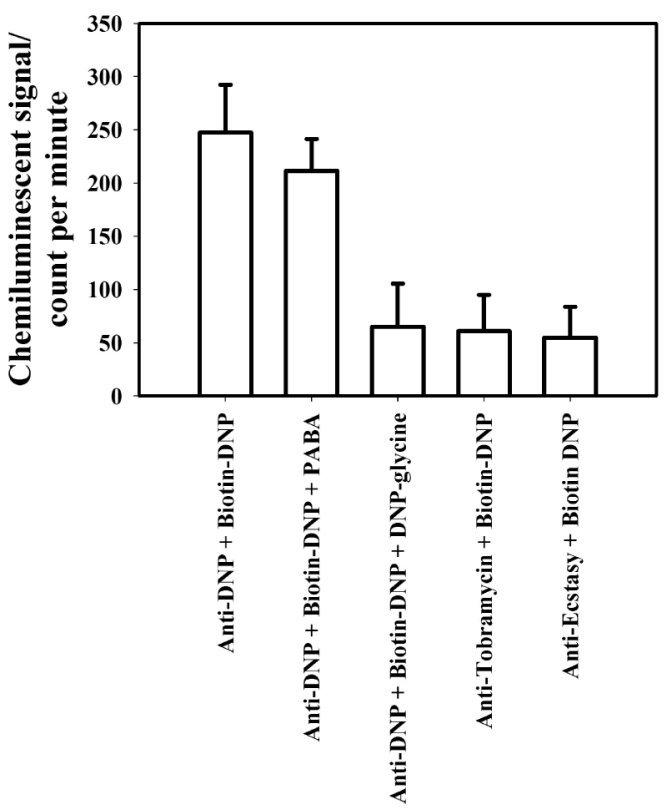

The reaction condition

Fig. 3 Specific interaction of anti-DNP antibody and biotinylated DNP based on luminol encapsulated liposomes as a signal generator. The microtiter plate was coated with anti-DNP antibody (Anti-DNP) or anti-Tobramycin antibody (Anti-Tobramycin) or anti-Ecstasy antibody (Anti-Esctasy). The reactions were carried out in the presence of biotinylated DNP (Biotin DNP), PABA, and/or DNP glycine.

Biotinylated, luminol encapsulated liposome was also used to detect the specific hybridization of oligonucleotides. The specific hybridization is shown in Fig. 5, where the chemiluminescence was inhibited only by the specific oligonucleotide strand with an LOD of $18 \mathrm{nM}$, and the signal-to-noise ratio $(S / N)$ was 11.69. The mismatched strand did not inhibit this reaction.

Thus, the biotinylated, luminol encapsulated liposome prepared and characterized here was shown to be potentially useful as signal generators to study specific antigen-antibody reactions and oligonucleotide hybridization.

Although the LODs of the biomolecule detection shown in this study were not lower than those of fluorescent encapsulated liposomes, which was about $1.7 \mathrm{pM},{ }^{22}$ it should be possible to improve the sensitivity of the system by various means $e . g$., optimization of the concentration of luminol in liposomes, the amount of biotin on liposome surface, the hybridization condition, etc.

\section{Conclusions}

Biotinylated liposomes with encapsulated chemiluminescent compound luminol have been prepared and characterized. The liposomes can be used as a signal generator to study specific antigen-antibody interactions and oligonucleotide hybridization. When biotinylated biorecognition molecules interact with their counterparts, streptavidin will serve as a bridge between the complexes with the luminol entrapped liposome signal generator. The system could be adapted to the lateral flow type of assay where the nitrocellulose membrane is used in place of a polystyrene microtiter plate. If proven successful, the generic

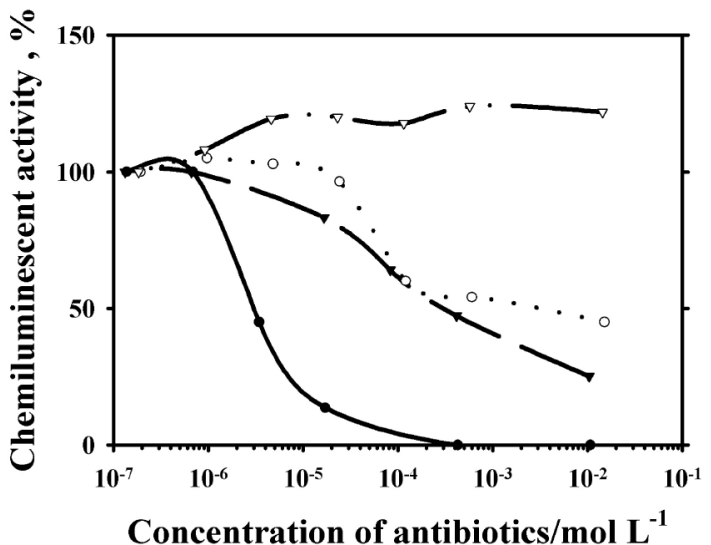

Fig. 4 Specific interaction of anti-Tobramycin antibody and biotinylated Tobramycin based on luminol encapsulated liposomes as a signal generator. The reactions were carried out in the presence of $\bullet$, Tobramycin; $\mathbf{\nabla}$, spectinomycin; $\bigcirc$, kanamycin; $\nabla$, amplicilin.

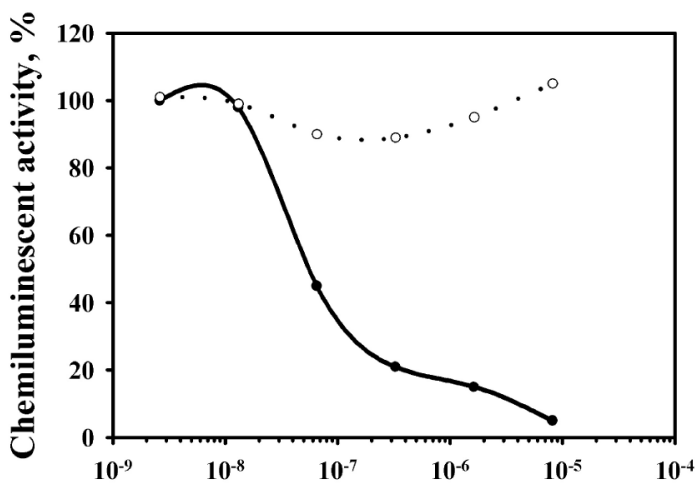

Concentration of oligonucleotides/mol $\mathrm{L}^{-1}$

Fig. 5 Specific hybridization of oligonucleotides based on luminol encapsulated liposomes as a signal generator. $\bullet$, Presence of synthetic $(+)$ strand; $\odot$, presence of mismatched strand.

liposome should be useful as a versatile signal generator for demonstrating specific interactions of biomolecules in these assays.

\section{Acknowledgements}

This study was supported by research grants from Chulabhorn Research Institute and from Thailand Research Fund (the Royal Golden Jubilee Scholarship). The authors thank Prof. Maurice Broughton for valuable suggestions.

\section{Supporting Information}

Supporting Information is available free of charge on the Web at http://www.jsac.or.jp/analsci/.

\section{References}

1. G. S. Rule, R. A. Montagna, and R. A. Durst, Clin. Chem., 1996, 42, 1206. 
2. M. C. Willis, B. Collins, T. Zhang, L. S. Green, D. P. Sebesta, C. Bell, E. Kellogg, S. C. Gill, A. Magallanez, S. Knauer, R. A. Bendele, P. S. Gill, and N. Janjić, Bioconjugate Chem., 1998, 9, 573.

3. S. Ahn-Yoon, T. R. DeCory, A. J. Baeumner, and R. A. Durst, Anal. Chem., 2003, 75, 2256.

4. C. H. Campbell, A. L. Miller, and L. H. Rome, Biochem. J., 1983, 214, 413.

5. J. A. Byrd, B. M. Hargis, D. E. Corrier, R. L. Brewer, D. J. Caldwell, R. H. Bailey, J. L. McReynolds, K. L. Herron, and L. H. Stanker, Poult. Sci., 2002, 81, 70.

6. T. Kamidate, M. Maruya, H. Tani, and A. Ishida, Anal. Sci., 2009, 25, 1163.

7. H. Chen, Y. Zheng, J. H. Jiang, H. L. Wu, G.-L. Shen, and R.-Q. Yu, Biosens. Bioelectron., 2008, 24, 684.

8. W. Zhan and A. J. Bard, Anal. Chem., 2007, 79, 459.

9. C.-H. Yoon, J.-H. Cho, H.-I. Oh, M.-J. Kim, C.-W. Lee, J.-W. Choi, and S.-H. Paek, Biosens. Bioelectron., 2003, 19, 289.

10. T. Suita, H. Tani, and T. Kamidate, Anal. Sci., 2000, 16, 527.

11. H. Arakawa, M. Maeda, and A. Tsuji, Anal. Biochem., 1979, 97, 248.

12. Y. Lv, Z. Zhang, D. He, and Y. Hu, Anal. Sci., 2003, 19, 625.

13. O. M. Steijger, D. A. Kamminga, A. Brummelhuis, and H. Lingeman, J. Chromatogr., A, 1998, 799, 57.

14. F. Wu, Y. Huang, and C. Huang, Biosens. Bioelectron., 2005, 21, 518 .

15. A. N. Díaz, M. C. R. Peinado, and M. C. T. Minguez, Anal. Chim. Acta, 1998, 363, 221.

16. E. H. White and M. M. Bursey, J. Am. Chem. Soc., 1964, 86,941 .

17. T. Suita and T. Kamidate, Anal. Sci., 1999, 15, 349.

18 A. K. Singh, P. K. Kilpatrick, and R. G. Carbonell,
Biotechnol. Prog., 1996, 12, 272.

19. A. L. Plant, M. V. Brizgys, L. L. Brown, and R. A. Durst, Anal. Biochem., 1989, 176, 420.

20. L. D. Leserman, J. Barbet, F. Kourilsky, and J. N. Weinstein, Nature, 1980, 288, 602.

21. H. Loughrey, M. B. Bally, and P. R. Cullis, Biochim. Biophys. Acta, 1987, 901, 157.

22. K. A. Edwards, K. L. Curtis, J. L. Sailor, and A. J. Baeumner, Anal. Bioanal. Chem., 2008, 391, 1689.

23. D. Martorell, S. T. Siebert, and R. A. Durst, Anal. Biochem., 1999, 271, 177.

24. A. K. Thompson, M. R. Mozafari, and H. Singh, Lait, 2007, 87, 349 .

25. G. P. Der-Balian, B. Gomez, R. S. Masino, and J. W. Parce, J. Immunol. Methods, 1990, 126, 281.

26. P. Corley and H. C. Loughrey, Biochim. Biophys. Acta, 1994, 1195, 149.

27. K. Kimura, Y. Arata, T. Yasuda, K. Kinosita, Jr., and M. Nakanishi, Biochim. Biophys. Acta, 1992, 1104, 9.

28. T. E. Redelmeier, J.-G. Guillet, and M. B. Ballyt, Drug Delivery, 1995, 2, 98.

29. A. D. Cooper, K. Balakrishnan, and H. M. McConnell, J. Biol. Chem., 1981, 256, 9379.

30. K. Kimura, Y. Arata, T. Yasuda, K. Kinosita, and M. Nakanishi, Immunology, 1990, 69, 323.

31. S. G. Stanton, A. B. Kantor, A. Petrossian, and J. C. Owicki, Biochim. Biophys. Acta, 1984, 776, 228.

32. K. Balakrishnan, S. Q. Mehdi, and H. M. McConnell, J. Biol. Chem., 1982, 257, 6434.

33. R. G. Coleman and K. A. Sharp, J. Mol. Biol., 2006, 362, 441.

34. A. K. Kenworthy, K. Hristova, D. Needham, and T. J. McIntosh, Biophys. J., 1995, 68, 1921.

35. Z. Gan and R. R. Marquardt, J. Biochem. Biophys. Methods, 1999, 39, 1 . 